\title{
INTEGRAÇÃO DE MÉTODOS QUANTITATIVOS E QUALITATIVOS DE PREVISÃO PARA DESENVOLVIMENTO DE UM SISTEMA DE PREVISÃO DE DEMANDA DE NOVOS PRODUTOS
}

\section{INTEGRATING QUANTITATIVE AND QUALITATIVE FORECASTING METHODS IN THE DEVELOPMENT OF A DEMAND FORECASTING SYSTEM FOR NEW PRODUCTS}

\author{
Fernando de Oliveira Lemos ${ }^{1}$; Flávio Sanson Fogliatto ${ }^{2}$ \\ ${ }^{1}$ Pontifícia Universidade Católica do Rio Grande do Sul - PUCRS - Porto Alegre - Brasil \\ fernando.lemos@pucrs.br \\ ${ }^{2}$ Universidade Federal do Rio Grande do Sul - UFRGS - Porto Alegre - Brasil \\ ffogliatto@producao.ufrgs.br
}

\begin{abstract}
Resumo
A integração de métodos qualitativos e quantitativos de previsão de demanda é uma alternativa para a obtenção de previsões mais acuradas, baseada na incorporação do conhecimento contextual nos processos matemáticos de previsão. Neste artigo, propõe-se uma metodologia para previsão de demanda de novos produtos através da integração de métodos qualitativos e quantitativos de previsão. A aplicabilidade da metodologia foi validada através de um estudo de caso. No estudo, detalhou-se o processo de previsão para um cenário de lançamento de um novo produto em um mercado estabilizado do setor de distribuição de energia elétrica. Utilizou-se o método de Analogia, integrando métodos de Extrapolação (Médias Móveis e Suavização Exponencial) com método de Opinião de Especialistas (Delphi).
\end{abstract}

Palavras-chave: previsão de demanda; integração de métodos; novos produtos; analogia.

\section{Introdução}

A integração de métodos de previsão é freqüentemente utilizada no intuito de melhorar a acurácia da previsão (BOPP, 1985). Previsões acuradas ajudam no desenvolvimento de estratégias, identificação de prioridades e alocação de recursos, além de permitir, pela operacionalização eficiente da produção, que as organizações ofereçam altos níveis de serviço aos clientes, forneçam informações de demandas futuras mais precisas aos fornecedores, planejem expansões de capacidade e evitem perdas nas vendas e estoques (KAHN, 2002).

A integração de métodos baseia-se em duas decisões (MACKAY; METCALFE, 2002): (i) a escolha dos métodos de previsão que serão integrados (métodos quantitativos, qualitativos ou ambos); e (ii) a seleção do método de integração (integração subjetiva ou matemática). 
Uma alternativa é a integração de métodos quantitativos e qualitativos, sintetizando os benefícios da precisão mecânica objetiva dos métodos matemáticos e habilidades interpretativas dos especialistas (WEBBY; O’CONNOR, 1996; GOODWIN, 2002). Diversos estudos apresentam evidências de que a integração, de métodos qualitativos e quantitativos em especial, é a melhor abordagem para a obtenção de previsões mais acuradas; ver, por exemplo, os trabalhos de Clemen (1989) e Blattberg e Hoch (1990).

O presente artigo apresenta uma proposta metodológica para estruturação de um sistema de previsão de demanda de novos produtos, baseado em integração de métodos qualitativos e quantitativos. Tal proposta alinha-se com uma área particularmente promissora para pesquisadores interessados em melhorar a prática de previsão: a identificação de condições específicas sobre as quais a integração de métodos quantitativos e qualitativos é mais útil.

A metodologia proposta é aplicada em um estudo de caso na indústria eletro-eletrônica. Mais especificamente, aborda-se o caso de um produto novo, para os quais existem poucos dados de demanda realizada, e onde a utilização de métodos qualitativos de previsão pode resultar em previsões mais acuradas.

O desenvolvimento da metodologia aqui apresentada foi motivado, num primeiro momento, por sua relevância acadêmica. Existe um número crescente de pesquisas acerca da integração de métodos qualitativos e quantitativos, conforme atesta o levantamento em Armstrong (2001). O desenvolvimento da metodologia justifica-se, também, por sua relevância prática, na medida em que a integração de métodos de previsão é uma das principais alternativas para a previsão de demanda por produtos novos, os quais não apresentam um histórico consolidado de demanda.

\section{Integração de métodos de previsão}

O sucesso no desenvolvimento de sistemas de suporte a tomada de decisões nas organizações tem produzido ambientes nos quais modelos matemáticos e inferências subjetivas podem ser facilmente integrados (WRIGHT; LAWRENCE; COLLOPY, 1996). Análises subjetivas devem ser integradas ao processo preditivo quando é importante evitar grandes erros nas previsões e em situações dinâmicas, onde os métodos quantitativos não conseguem agregar aos modelos matemáticos mudanças internas e externas ao ambiente organizacional (GOODWIN, 2000; ARMSTRONG, 2001).

As previsões obtidas por métodos qualitativos podem ser baseadas em conhecimento contextual (experiência do especialista na indústria, familiaridade com os produtos/serviços a serem previstos e conhecimento do mercado) e/ou conhecimento técnico (conhecimento de métodos de previsão e análise de dados) (WEBBY; O’CONNOR, 1996). Previsões mais acuradas são obtidas pela integração de métodos quantitativos com previsões qualitativas baseadas em conhecimento 
contextual (SANDERS; RITZMAN, 1995).

A vantagem da integração de métodos quantitativos com qualitativos é a incorporação do domínio de conhecimento (conhecimento sobre a natureza e comportamento das variáveis de interesse) no processo preditivo. O domínio de conhecimento é resultado da interpretação da informação contextual pelo especialista em previsão, da sua habilidade de captar o significado apropriado da informação contextual. A informação contextual pode ser obtida de séries temporais, de fontes de informações públicas e/ou de fontes internas da organização (WEBBY; O'CONNOR, 2001).

A integração dos métodos de previsão pode se dar de quatro maneiras não mutuamente exclusivas (WEBBY; O’CONNOR, 1996): (i) ajuste subjetivo; (ii) decomposição de séries temporais; (iii) combinação de previsões; e (iv) desenvolvimento de um modelo de previsão. A escolha do melhor método de integração dependerá das condições específicas de uma determinada situação (GOODWIN, 2002).

O Ajuste Subjetivo agrega informação contextual na previsão quantitativa (Figura 1), informação que o modelo matemático geralmente não considera ou que a série temporal não inclui. Esta alternativa de integração é geralmente a mais fácil de implementar e a que apresenta o melhor custo/benefício. Entretanto, o ajuste subjetivo pode introduzir informações tendenciosas na previsão se o especialista não traduzir corretamente os fatores contextuais para o processo preditivo (WEBBY; O’CONNOR, 1996).

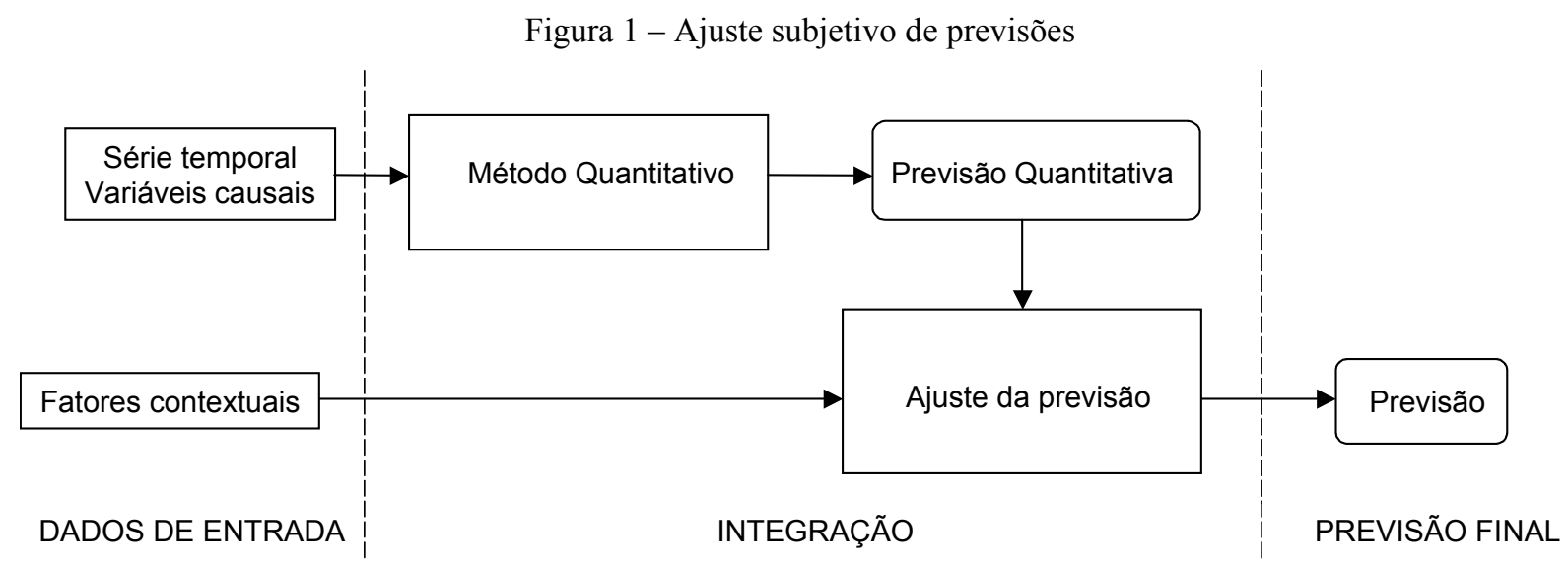

Fonte: Webby e O’Connor (1996)

Deve-se usar ajuste subjetivo de previsões estatísticas (WRIGHT; LAWRENCE; COLLOPY, 1996; SANDERS; RITZMAN, 2001): (i) quando existe um grande conhecimento sobre as variáveis a serem preditas e sobre mudanças que afetarão estas variáveis; (ii) em situações com alto grau de incerteza; e (iii) quando informações contextuais invalidam a suposição de constância dos padrões de demanda passados. 


\section{Previsão de novos produtos}

A disponibilidade de dados para previsão de demanda e a seleção dos métodos utilizados dependem diretamente da maturidade do produto; conseqüentemente, o estágio do ciclo de vida do produto é determinante no processo preditivo (ARMSTRONG; BRODIE, 1999). O ciclo de vida de um produto pode ser dividido em cinco fases (CHAMBERS; MULLICK; SMITH, 1971): (i) de desenvolvimento; (ii) de testes e introdução no mercado; (iii) de crescimento rápido no mercado; (iv) de estabilidade no mercado; e (v) de declínio.

No estágio de desenvolvimento de produtos a seleção dos métodos depende do mercado e da concepção do produto. Para um mercado estabelecido deve-se primeiramente comparar o produto proposto com similares de concorrentes ou da própria organização através da utilização de uma equipe multidisciplinar. Os métodos quantitativos são utilizados nesta fase quando é possível obter informações (séries históricas e variáveis causais) de produtos análogos (ARMSTRONG; BRODIE, 1999).

O método de previsões por Analogia (Figura 2) extrapola resultados de situações análogas para predizer uma situação de interesse (ARMSTRONG; BRODIE, 1999). Na utilização deste método tenta-se identificar um produto similar, cujo padrão de penetração no mercado seja similar ao do novo produto analisado (CHAMBERS; MULLICK; SMITH, 1971). Para evitar o viés no julgamento por parte dos especialistas do processo de previsão (HOGARTH; MAKRIDAKIS, 1981), necessita-se de um método estruturado para comparação análoga (SOUDER; THOMAS, 1994).

Figura 2 - Integração entre métodos de previsão (linhas pontilhadas indicam possíveis relações)

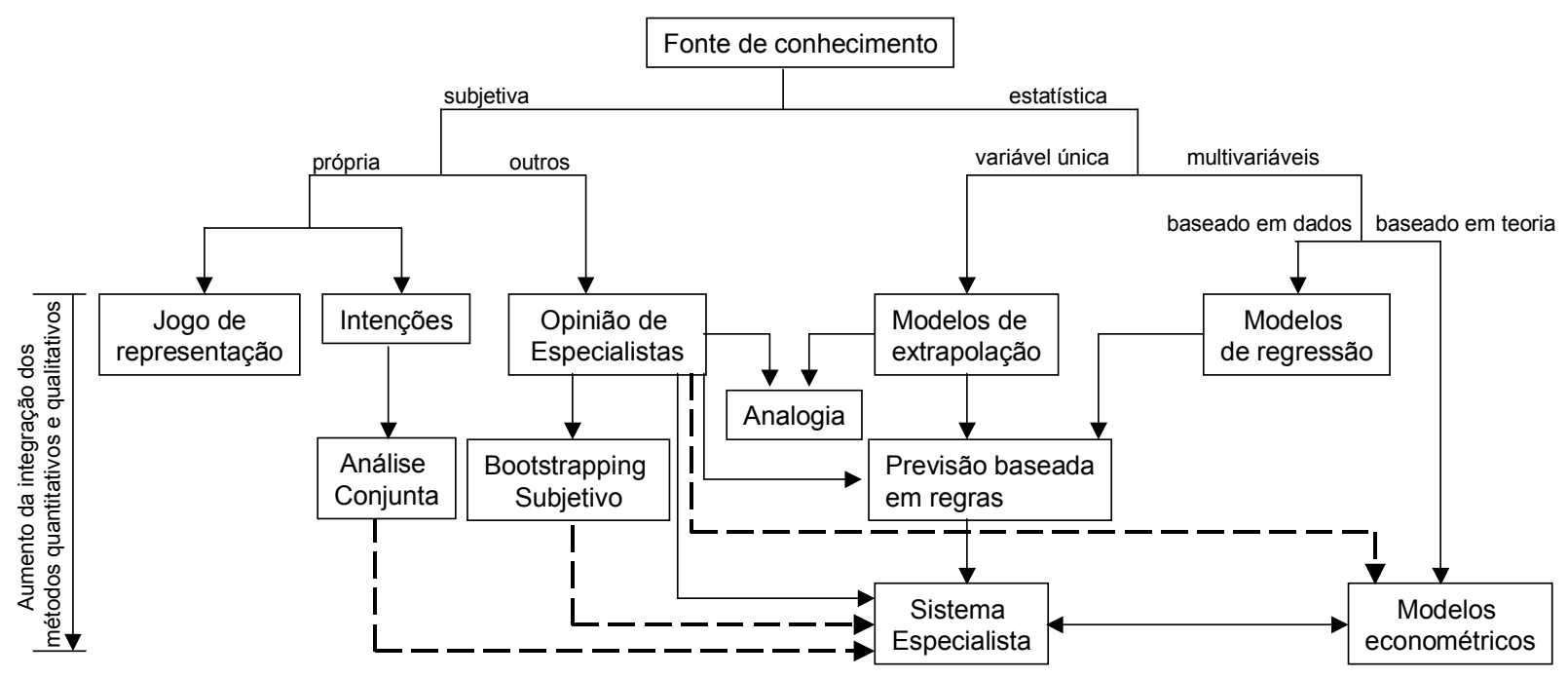

Fonte: Adaptado de Armstrong e Brodie (1999) 
O método é operacionalizado através da análise de características em comum entre o produto de interesse e o produto similar (ou conjunto de produtos similares), como, por exemplo: funcionalidades dos produtos, classe de consumidores, estrutura do mercado (potencial de mercado, demanda histórica, concorrentes, etc) e nível de inovação (SOUDER; THOMAS, 1994). Uma vez definidas as características similares, obtêm-se, baseado no comportamento passado destas características nos produtos similares, estimativas de demanda para o novo produto. A análise de dados utiliza métodos de Extrapolação de séries temporais integrados com métodos baseados em Opiniões de Especialistas conforme apresentado na Figura 2 (ARMSTRONG; BRODIE, 1999).

\section{Metodologia proposta e estudo de caso}

A metodologia proposta para previsão de demanda de novos produtos é operacionalizada em seis etapas: (i) Definição do problema; (ii) Obtenção de dados; (iii) Escolha do(s) método(s) de previsão; (iv) Implementação do(s) método(s); e (v) Validação do(s) método(s). As etapas são detalhadas na seqüência. Em paralelo ao detalhamento da metodologia, apresenta-se a sua aplicação em um estudo de caso no setor eletro-eletrônico. Optou-se por esta estratégia de apresentação para evitar redundâncias e objetivar a apresentação da metodologia.

\subsection{Definição do problema}

O estudo de caso foi desenvolvido em uma empresa que produz medidores de energia elétrica. Os produtos de interesse neste estudo são os medidores eletrônicos de energia elétrica, que foram desenvolvidos para substituir os medidores eletromecânicos fabricados pela empresa, especialmente na classe de consumidores residenciais das distribuidoras de energia elétrica. Previsões de demanda desses medidores no mercado brasileiro são essenciais para planejamentos tático e estratégico da empresa, sendo utilizadas para o planejamento agregado de produção, dimensionamento da capacidade produtiva e alocação de recursos em P\&D.

Definiu-se como problema a ser resolvido a previsão de demanda de medidores eletrônicos (todos os modelos agregados) para o mercado interno. Estas previsões têm sido superestimadas pelos especialistas da empresa, dificultando o planejamento de produção de curto e médio prazo. Como a empresa estava se baseando em uma estimativa de demanda errônea para a programação de produção, gerava estoques desnecessários de matéria-prima.

As previsões dos especialistas consideravam que a substituição de medidores se concentraria nos consumidores residenciais de energia, que são os consumidores que demandam maior quantidade de medidores. A substituição de medidores na classe residencial de consumo depende, além do interesse das distribuidoras, do interesse dos órgãos reguladores do setor. Uma mudança de cenário pode afetar diretamente a demanda pelo novo produto, como, por exemplo, a mudança de 
leis sobre medição de energia em nível residencial. O objetivo deste estudo não é a previsão de cenários e sim a previsão de demanda do produto dentro do atual cenário de mercado e considerando a continuidade deste cenário no curto e médio prazo.

Um período de previsão mensal foi definido em função da unidade de previsão requerida para as tomadas de decisão quanto ao planejamento de produção da empresa e dimensionamento de estoques de matérias-primas. O intervalo entre previsões foi definido como de 6 meses. Foi considerado que não havia necessidade de alocação de recursos para revisões freqüentes das previsões até que os produtos entrassem no ciclo de crescimento rápido no mercado. Como os produtos estão em uma fase do ciclo de vida influenciada por numerosos fatores contextuais, um horizonte de previsão de 6 meses foi estipulado nesse processo preditivo.

\subsection{Obtenção de dados}

A estrutura do mercado deve ser identificada completamente para a previsão de demanda de novos produtos. No caso de previsões de vendas do novo produto não há dados históricos diretos para a previsão através de projeções de tendência ou análise causal. Entretanto, outras formas de dados primários e secundários estão disponíveis e, na extensão que os recursos permitem, utilizamse análises subjetivas na tomada de decisão sobre o novo produto.

A organização disponibilizou informações contextuais sobre o mercado em que estão inseridos os medidores eletrônicos e o histórico de demanda do produto similar (medidores eletromecânicos polifásicos). As informações contextuais foram coletadas em fontes independentes (literatura e especialistas do setor de distribuição de energia elétrica). A confiabilidade e relevância dos dados para o processo preditivo foram confirmadas pelos especialistas da empresa. As informações contextuais foram utilizadas para a aplicação do método qualitativo de previsão e a série histórica análoga para a implementação de métodos quantitativos.

O mercado de distribuição de energia elétrica é atendido por 64 concessionárias, estatais ou privadas, em todo o país. São atendidos cerca de 47 milhões de unidades consumidoras, das quais 85\% são consumidores residenciais (ANEEL, 2005).

Os medidores eletrônicos têm sido usados preferencialmente em consumidores comerciais e industriais no Brasil. A demanda por este tipo de medidor é função da necessidade de discriminação de consumo por segmento de horário (ponta e fora de ponta), de energia consumida e de demanda máxima verificada. Essa estratificação do consumo permite diferentes métodos de taxação através da mensuração de uso de cargas, horário de uso ou pico de carga.

O estudo de caso focou na estimativa da taxa de substituição deste tipo de medidor pelo novo produto e de valores pontuais mensais de demanda de medidores eletrônicos. A série temporal dos medidores polifásicos foi analisada graficamente para identificação de erros de leitura e para 
localizar dados atípicos decorrentes de eventos especiais passados e não sistemáticos.

\subsection{Escolha do(s) método(s) de previsão}

A escolha do método de Analogia é justificada pelo fato dos medidores eletrônicos e eletromecânicos possuírem funcionalidades semelhantes e o mesmo mercado consumidor. O método de Analogia utiliza, para a análise dos dados e previsão de demanda, métodos de Extrapolação de séries temporais integrados com métodos baseados em Opiniões de Especialistas. A empresa não mostrou restrições a investimentos no seu sistema de previsão de demanda, podendo assim ser implementados métodos de Extrapolação complexos. A análise gráfica realizada na série temporal dos medidores eletromecânicos polifásicos não indicou sazonalidades ou tendências aparentes, mas como graficamente não podíamos concluir sobre a existência destes componentes do padrão de demanda optou-se pela implementação de todos os métodos de Extrapolação Simples: Média Móvel e Suavização Exponencial (Simples, Linear de Holt e de Holt-Winters), e uma posterior escolha do método de Extrapolação mais adequado para a situação estudada. Características importantes destes métodos podem ser avaliadas em Makridakis, Wheelwright e Hyndman (1998).

Os métodos de Extrapolação foram utilizados para obter previsões de demanda dos medidores polifásicos, para os quais existem séries históricas disponíveis, e o método Delphi, baseado em opiniões de especialistas, para estimar o percentual de substituição no mercado dos medidores eletromecânicos pelo seu análogo eletrônico. As previsões dos métodos de Extrapolação para as séries análogas serão ajustadas subjetivamente pelas previsões obtidas com o método Delphi para a obtenção das previsões finais de demanda.

\subsection{Implementação do(s) método(s) de previsão}

Para um novo produto lançado em um mercado estabelecido, como no caso em estudo, deve-se primeiramente comparar o produto proposto com similares de concorrentes ou da própria organização através da utilização de uma equipe multidisciplinar. O mapeamento do domínio de conhecimento de um produto existente foi utilizado para definir inferências sobre o novo produto. Os dados de demanda relativos aos medidores eletromecânicos foram utilizados para a implementação do método de Analogia (método de Extrapolação integrado ao método de Opiniões de Especialistas).

A implementação do método de Analogia seguiu os seguintes passos: (i) implementação do método Delphi (para a obteção das opiniões de especialistas); (ii) implementação dos métodos de Extrapolação; e (iii) integração dos métodos. 


\subsubsection{Implementação do método Delphi}

A implementação do método Delphi foi realizada em seis etapas (Wright; Giovinazzo, 2000): (i) definição dos respondentes e elaboração do questionário; (ii) validação do questionário a ser aplicado; (iii) aplicação do questionário; (iv) tabulação e análise dos dados; (v) nova rodada de aplicação do questionário; e (vi) obtenção das previsões finais. O objetivo do Delphi era obter informações sobre taxa de substituição dos medidores para ajuste das previsões quantitativas dos medidores eletromecânicos.

A amostra de respondentes foi definida em função da disponibilidade das distribuidoras (concessionárias) de energia em responder o questionário. Foram convidadas a participar da pesquisa 4 distribuidoras, mas somente 2 distribuidoras e a empresa fornecedora do medidor participaram. Cada empresa determinou um especialista para participar da aplicação do Delphi, o que prejudicou a análise devido a pequena amostra de especialistas, mas facilitou a obtenção de consenso sobre a previsão requerida. A pesquisa também buscou uma aproximação entre as empresa fornecedora e seus clientes, para um melhor entendimento das intenções e do comportamento dos mesmos.

O questionário foi elaborado com base nas informações de mercado obtidas com especialistas da empresa e em literatura especializada de pesquisa de mercado (CHURCHILL, 2001; MALHOTRA, 2001; MESQUITA; SANTORO, 2004). Com a aplicação do questionário foram obtidas informações sobre o mercado e sobre a substituição dos medidores eletromecânicos por medidores eletrônicos.

Analisando as taxas de substituição fornecidas pelos respondentes (Tabela 1) notou-se um otimismo da empresa fornecedora quanto à intenção de compra dos medidores eletrônicos para substituição dos medidores eletromecânicos. As taxas de substituição informadas pelos especialistas diferiram quanto aos seus valores, o que gerou a necessidade de uma nova rodada do questionário para a obtenção do consenso. As concessionárias têm intenções de substituição bem menores do que as vislumbradas pela empresa fornecedora. Os valores das estimativas foram tabulados (previsões médias foram geradas) e retornados para a segunda rodada do Delphi, juntamente com as justificativas fornecidas para as estimativas da primeira rodada.

Tabela 1 - Taxas estimadas de substituição dos medidores eletromecânicos polifásicos pelos eletrônicos na primeira rodada do Delphi

\begin{tabular}{cccc}
\hline & $\begin{array}{c}\text { Cenário muito pouco } \\
\text { favorável de mercado }\end{array}$ & $\begin{array}{c}\text { Cenário em condições } \\
\text { atuais de mercado }\end{array}$ & $\begin{array}{c}\text { Cenário muito favorável } \\
\text { de mercado }\end{array}$ \\
\hline Distribuidora A & $2 \%$ & $3 \%$ & $4,5 \%$ \\
Distribuidora B & $0,5 \%$ & $1 \%$ & $100 \%$ \\
Empresa & $5 \%$ & $10 \%$ & $100 \%$ \\
\hline Média & $\mathbf{2 , 5 \%}$ & $\mathbf{4 , 7 \%}$ & $\mathbf{6 8 , 2 \%}$ \\
\hline
\end{tabular}


O objetivo da segunda rodada era revisar as respostas da primeira baseando-se nas novas informações disponibilizadas pelos outros especialistas. Assim, foi dada aos respondentes a oportunidade de alterar suas estimativas iniciais com base no feedback fornecido. Com a segunda rodada de aplicação do questionário obteve-se consenso para algumas estimativas. Os resultados da segunda rodada são apresentados na Tabela 2. Outras apresentaram valores muito próximos; foram consideradas, assim, as médias dos valores. Os valores que apresentaram grandes diferenças não influenciavam os objetivos deste estudo; desta forma, a aplicação do método Delphi foi finalizada após a segunda rodada.

Tabela 2 - Taxas estimadas de substituição dos medidores eletromecânicos polifásicos pelos eletrônicos na segunda

\begin{tabular}{cccc}
\multicolumn{4}{c}{ rodada do Delphi } \\
\hline & $\begin{array}{c}\text { Cenário muito pouco } \\
\text { favorável de mercado }\end{array}$ & $\begin{array}{c}\text { Cenário em condições } \\
\text { atuais de mercado }\end{array}$ & $\begin{array}{c}\text { Cenário muito favorável } \\
\text { de mercado }\end{array}$ \\
\hline Distribuidora A & $2 \%$ & $3 \%$ & $68,1 \%$ \\
Distribuidora B & $2,5 \%$ & $3 \%$ & $100 \%$ \\
Empresa & $2,5 \%$ & $4,7 \%$ & $100 \%$ \\
\hline Média & $\mathbf{2 , 3 \%}$ & $\mathbf{3 , 6 \%}$ & $\mathbf{8 9 , 5 \%}$ \\
\hline
\end{tabular}

Os valores de maior interesse são as taxas de substituição dos polifásicos para os cenários atual e muito pouco favorável de demanda dos medidores eletrônicos na classe de consumidores residenciais. Somente estes dois cenários serão abordados devido ao horizonte de previsão de médio prazo considerado neste estudo de caso e no qual não se esperam favorecimentos de fatores contextuais para uma maior demanda de medidores eletrônicos.

O tempo de implementação do método foi de aproximadamente 2 meses, pois apesar da complexidade do tema, o número de respondentes engajados na pesquisa era limitado e o domínio de conhecimento, destes especialistas, muito grande.

\subsubsection{Implementação dos métodos de extrapolação}

Com a utilização de um software de previsão foi possível implementar os métodos de Extrapolação. As séries temporais foram divididas em duas partes, uma parte para o ajuste do modelo matemático (39 períodos mensais) e a outra para validação do método ( 6 últimos períodos mensais), em função do horizonte de previsão definido.

A primeira análise focou no período ideal para a média móvel. Desta forma, comparou-se a média dos erros percentuais absolutos (MAPE) dos valores ajustados em relação aos valores da série histórica para Médias Móveis com diferentes períodos $(\mathrm{n}=1,2,3,4,5$ e 6). O período com menor MAPE $(0,1953)$ correspondeu a $n=3$, sendo este utilizado para comparações com o método de Suavização Exponencial (Tabela 3). 
Tabela 3 - MAPE para o método de Média Móvel com diferentes períodos

\begin{tabular}{ccccccc}
\hline Média Móvel & $\mathrm{n}=1$ & $\mathrm{n}=2$ & $\mathrm{n}=3$ & $\mathrm{n}=4$ & $\mathrm{n}=5$ & $\mathrm{n}=6$ \\
\hline MAPE & 0,1956 & 0,2075 & 0,1953 & 0,1964 & 0,2106 & 0,2131 \\
\hline
\end{tabular}

As modelagens da série temporal de polifásicos (obtidas com o software) vêm apresentadas na Figura 3, conjuntamente com as previsões pontuais e o intervalo de confiança para a previsão (nível de confiança $=95 \%$ ). O método de Suavização Exponencial (sem tendência e com sazonalidade multiplicativa) apresentou um MAPE de 0,1832 e um ajuste deficiente, com valor muito baixo de R2 $(0,1539)$. As previsões obtidas com a implementação dos métodos quantitativos e os limites inferior e superior do intervalo de confiança são apresentados na Tabela 4.

Figura 3 - Modelagens para as séries temporais do medidor eletromecânico polifásico pelo (a) método de Média Móvel e pelo (b) método de Suavização Exponencial.

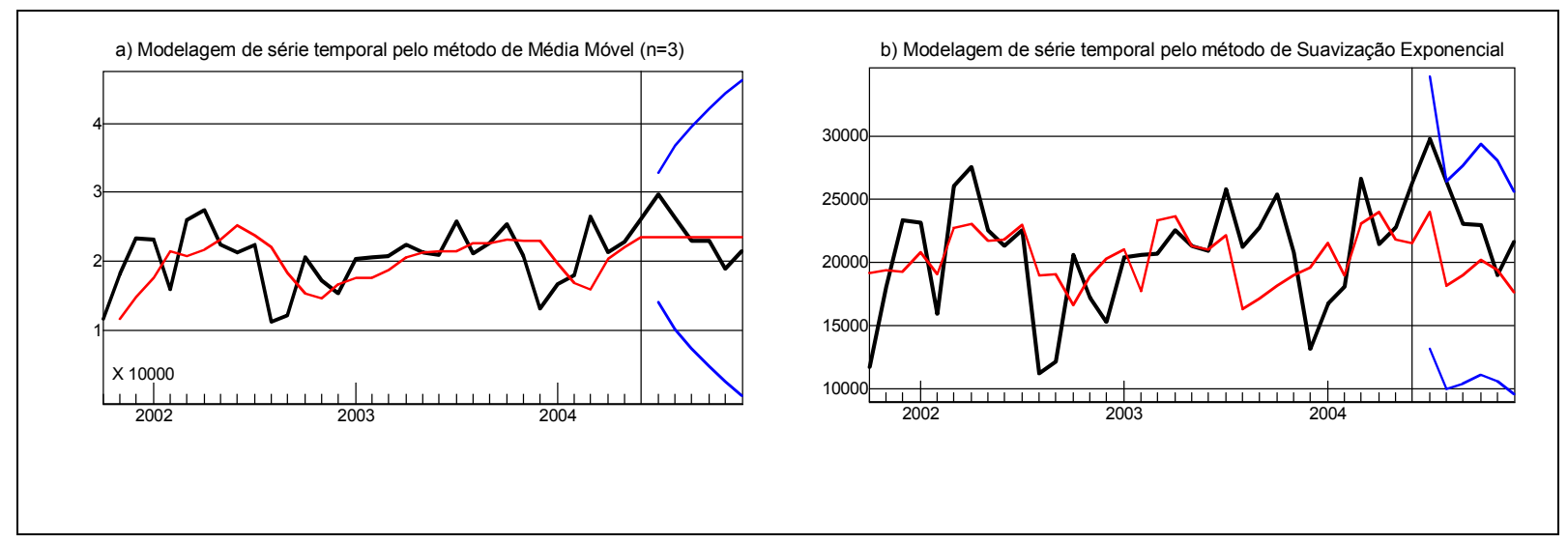

Tabela 4 - Demandas previstas (em unidades de produto), limites inferior e limites superior (nível de confiança = 95\%) para o medidor eletromecânico polifásico

\begin{tabular}{lllllll}
\hline \multicolumn{1}{c}{ Método } & \multicolumn{3}{c}{ Média Móvel } & \multicolumn{3}{c}{ Suavização Exponencial } \\
\hline \multirow{2}{*}{ Período } & $\begin{array}{l}\text { Limite } \\
\text { inferior }\end{array}$ & $\begin{array}{l}\text { Previsão } \\
\text { pontual }\end{array}$ & $\begin{array}{l}\text { Limite } \\
\text { superior }\end{array}$ & $\begin{array}{l}\text { Limite } \\
\text { inferior }\end{array}$ & $\begin{array}{l}\text { Previsão } \\
\text { pontual }\end{array}$ & $\begin{array}{l}\text { Limite } \\
\text { superior }\end{array}$ \\
\hline julho-04 & 14.871 & 24.275 & 33.6643 & 13.116 & 23.916 & 34.716 \\
agosto-04 & 10.983 & 24.275 & 37.531 & 9.959 & 18.174 & 26.390 \\
setembro-04 & 8.000 & 24.275 & 40.514 & 10.409 & 19.004 & 27.598 \\
Outubro-04 & 5.485 & 24.275 & 43.029 & 11.069 & 20.213 & 29.358 \\
novembro-04 & 3.269 & 24.275 & 45.245 & 10.576 & 19.327 & 28.079 \\
dezembro-04 & 1.266 & 24.275 & 47.248 & 9.636 & 17.628 & 25.620 \\
\hline
\end{tabular}

\subsubsection{Integração dos métodos}

As previsões finais foram obtidas através da integração do Delphi e dos métodos de Extrapolação. Utilizou-se um ajuste da previsão quantitativa (valores da Tabela 4): os valores de previsão pontual da Tabela 4 foram multiplicados pelos percentuais médios de substituição (cenários atual e muito pouco favorável) dos medidores polifásicos (Tabela 2). As previsões pontuais finais dos medidores eletrônicos são apresentadas na Tabela 5. 


\subsection{Validação do(s) método(s) de previsão}

As previsões obtidas pelo método de Analogia para os dois cenários foram comparadas com as previsões dos especialistas da empresa. Os métodos foram avaliados através da acurácia das previsões para um horizonte de 6 meses. Utilizou-se a média dos erros percentuais absoluto (MAPE) e o erro percentual absoluto (APE) como medidas de acurácia dos métodos.

Tabela 5 - Previsões de demandas pontuais finais em unidades de medidores eletrônicos por cenário

\begin{tabular}{lrccc}
\hline \multicolumn{1}{c}{ Método } & & Média Móvel & \multicolumn{2}{c}{ Suavização Exponencial } \\
\hline \multicolumn{1}{c}{ Cenários } & Atual & Muito pouco favorável & Atual & Muito pouco favorável \\
\hline julho-04 & 873 & 558 & 861 & 550 \\
agosto-04 & 873 & 558 & 654 & 418 \\
setembro-04 & 873 & 558 & 684 & 437 \\
outubro-04 & 873 & 558 & 728 & 465 \\
novembro-04 & 873 & 558 & 696 & 445 \\
dezembro-04 & 873 & 558 & 635 & 405 \\
\hline
\end{tabular}

Os resultados da análise de acurácia das previsões com taxa de substituição para o cenário atual foram comparados com a acurácia das previsões com taxa de substituição para o cenário muito pouco favorável. Analisando os resultados, o menor MAPE $(5,5087)$ foi obtido para o método de Suavização Exponencial ajustado com a taxa de substituição para o cenário muito pouco favorável (Tabelas 6 e 7).

Tabela 6 - Acurácia das demandas pontuais dos medidores eletrônicos para o cenário atual

\begin{tabular}{lll|l|llll}
\hline \multicolumn{1}{c}{ Método } & \multicolumn{3}{c}{ Média Móvel } & \multicolumn{3}{c}{ Suavização Exponencial } \\
\hline Período & $\begin{array}{l}\text { Demanda } \\
\text { real }\end{array}$ & $\begin{array}{l}\text { Previsão } \\
\text { pontual }\end{array}$ & APE & MAPE & $\begin{array}{l}\text { Previsão } \\
\text { pontual }\end{array}$ & APE & MAPE \\
\hline julho-04 & 44 & 873 & 18,8466 & & 861 & 18,5676 & \\
agosto-04 & 178 & 873 & 3,9059 & & 654 & 2,6756 & \\
setembro-04 & 744 & 873 & 0,1737 & 10,8811 & 684 & 0,0805 & 8,9991 \\
outubro-04 & 79 & 873 & 10,0538 & & 728 & 8,2110 & \\
novembro-04 & 40 & 873 & 20,8313 & & 696 & 16,3943 & \\
dezembro-04 & 70 & 873 & 11,4750 & & 635 & 8,0658 & \\
\hline
\end{tabular}

Tabela 7 - Acurácia das demandas pontuais dos medidores eletrônicos para o cenário muito pouco favorável

\begin{tabular}{llllllll}
\hline \multicolumn{1}{c}{ Método } & & \multicolumn{3}{c}{ Média Móvel } & \multicolumn{2}{c}{ Suavização Exponencial } \\
\hline Período & $\begin{array}{l}\text { Demanda } \\
\text { real }\end{array}$ & $\begin{array}{l}\text { Previsão } \\
\text { pontual }\end{array}$ & APE & MAPE & $\begin{array}{l}\text { Previsão } \\
\text { pontual }\end{array}$ & APE & MAPE \\
\hline julho-04 & 44 & 558 & 11,6798 & & 550 & 11,5015 & \\
agosto-04 & 178 & 558 & 2,1343 & & 418 & 1,3483 & \\
setembro-04 & 744 & 558 & 0,2501 & 6,6741 & 437 & 0,4125 & 5,5087 \\
outubro-04 & 79 & 558 & 6,0622 & & 465 & 4,8848 & \\
novembro-04 & 40 & 558 & 12,9478 & & 445 & 10,1130 & \\
dezembro-04 & 70 & 558 & 6,9702 & & 405 & 4,7921 & \\
\hline
\end{tabular}

As previsões obtidas com o método de Suavização Exponencial para o cenário muito pouco favorável foram comparadas com as previsões dos especialistas da empresa conforme apresentado na Tabela 8. Os valores de APE e MAPE evidenciam os erros obtidos com o método de previsão selecionado pela metodologia proposta, o que é justificado pela incerteza inerente ao processo de 
previsão de novos produtos.

O método selecionado apresentou menor MAPE que o obtido para as previsões dos especialistas da empresa. Apesar do erro obtido com o método de Analogia ser grande, o método oferece previsões com menor erro que as dos especialistas da empresa. A vantagem de utilização do método de Analogia foi a utilização do método qualitativo (Delphi), o qual disponibilizou informações contextuais de suma importância para o processo preditivo.

Tabela 8 - Acurácia das demandas pontuais previstas pelos especialistas da empresa e pelo método selecionado para os medidores eletrônicos

\begin{tabular}{llllllll}
\hline \multicolumn{1}{c}{ Método } & \multicolumn{3}{c}{ Suavização Exponencial } & \multicolumn{3}{c}{ Especialistas } \\
\hline Período & $\begin{array}{l}\text { Demanda } \\
\text { real }\end{array}$ & $\begin{array}{l}\text { Previsão } \\
\text { pontual }\end{array}$ & APE & MAPE & $\begin{array}{l}\text { Previsão } \\
\text { pontual }\end{array}$ & APE & MAPE \\
\hline julho-04 & 44 & 550 & 11,5015 & & 2.000 & 44,4545 \\
agosto-04 & 178 & 418 & 1,3483 & & 3.000 & 15,8539 & \\
setembro-04 & 744 & 437 & 0,4125 & 5,5078 & 2.700 & 2,6290 & 30,6639 \\
outubro-04 & 79 & 465 & 4,8848 & & 3.000 & 36,9747 & \\
novembro-04 & 40 & 445 & 10,1130 & & 700 & 16,5000 & \\
dezembro-04 & 70 & 405 & 4,7921 & & 4.800 & 67,5714 & \\
\hline
\end{tabular}

\section{Conclusão}

Esse trabalho teve como objetivo principal a apresentação de uma metodologia para previsão de demanda de novos produtos com integração de métodos qualitativos e quantitativos de previsão. A aplicabilidade da metodologia proposta foi validada através de um estudo de caso, onde detalhouse o processo de previsão para um cenário de lançamento de um novo produto em um mercado estabilizado do setor de distribuição de energia elétrica (isto é, para um produto apresentando um padrão de demanda irregular).

A aplicação da metodologia para o produto no estágio de teste e introdução no mercado resultou na utilização do método de Analogia, integrando métodos de Extrapolação (Médias Móveis e Suavização Exponencial) com método de Opinião de Especialistas (Delphi). Utilizaram-se dados de demanda histórica de produtos similares ao lançado no mercado e informações contextuais sobre o novo produto e seu mercado potencial. A precisão das previsões resultou em baixa acurácia. Entretanto, apesar do grande erro das previsões com o método selecionado, estas apresentaram menor erro que as dos especialistas da empresa.

A pesquisa Delphi apresentou evidências de respostas tendenciosas. Apesar de um dos princípios do método ser a eliminação do domínio de um especialista sobre o processo preditivo, o mesmo não prevê que os respondentes possam não estar motivados a diversas rodadas de aplicação do questionário. No estudo de caso apresentado, observou-se um desejo dos especialistas em encontrar a previsão consensual rapidamente. Apesar desse fator pesar nos resultados, as previsões obtidas foram mais acuradas (se comparadas àquelas obtidas pelo método puramente quantitativo), 
favorecendo o planejamento tático e estratégico da empresa.

A melhoria de acurácia das previsões com o método proposto pode ser traduzida em melhorias no planejamento do processo produtivo dos medidores eletrônicos, principalmente na compra de matéria-prima, programação de produção e alocação de mão-de-obra. Previsões mais precisas poderiam, possivelmente, ser obtidas com a aplicação do Delphi com uma amostra maior de respondentes. A amostra limitou a pesquisa Delphi em exploratória, não podendo ser considerada uma pesquisa conclusiva sobre o assunto abordado.

\begin{abstract}
The integration of qualitative and quantitative forecasting methods is an approach based on the incorporation of contextual knowledge in mathematical forecasting processes. Such approach may lead to more accurate demand forecasts. In this paper we propose a methodology to forecast the demand for new products based on the integration of qualitative and quantitative forecasting methods. The proposed methodology is validated in a case study from the electronics industry. In the case study we detail the forecasting process of a new product to be introduced in a stable market, in the electricity distribution sector. To forecast the new product demand we use forecasting by analogy, integrating extrapolation methods (moving averages and exponential smoothing techniques) with expert opinion gathered through the Delphi method.
\end{abstract}

Key-words: demand forecast; integration of methods; new products; forecasting by analogy.

\title{
Referências
}

ANEEL. Informações Técnicas. Disponível em: <http://www.aneel.gov.br/48.htm> Acesso em: 10 jan. 2005.

ARMSTRONG, J. Selecting Forecasting Methods. In: ARMSTRONG, J. Principles of forecasting: a handbook for researchers and practitioners. Boston: Kluwer Academic Publishers, 2001.

ARMSTRONG, J. S.; BRODIE, R. J. Forecasting for marketing. In: HOOLEY, G. J.; HUSSEY, M. K. Quantitative methods in marketing. 2. ed., London: International Thompson Business Press, 1999.

BLATTBERG, R. C.; HOCH, S. J. Database models and managerial intuition: $50 \%$ model $+50 \%$ manager. Management Science. v. 36, n. 8, p. 887-899, 1990.

cross ref

BOPP, A. E. On combining forecasts: some extensions and results. Management Science. v. 31, n. 12, p. 1492-1498, 1985.

CHAMBERS, J. C.; MULLICK, S. K.; SMITH, D. D. How to choose the right forecasting technique. Harvard Business Review. v. 49, p. 45-57, jul.-ago. 1971.

CHURCHILL, G. A. Basic marketing research. 4. ed., New York: The Dryden Press, 2001.

CLEMEN, R. T. Combining forecasts: a review and annotated bibliography. International Journal of Forecasting. v. 5, n. 4, p. 559-583, 1989.

cross ${ }^{\text {ref }}$

GOODWIN, P. Integrating management judgment and statistical methods to improve short-term forecasts. Omega. v. 30, n. 2, p. 127-135, 2002.

cross ${ }^{\text {ref }}$

GOODWIN, P. Improving the voluntary integration of statistical forecasts and judgment. International Journal of 
HOGARTH, R.M.; MAKRIDAKIS, S. Forecasting and planning: an evaluation. Management Science. v. 27, n. 2, p. 393-404, 1981.

cross ref

KAHN, K. An exploratory investigation of new product forecasting practices. The Journal of Product Innovation Management. v. 19, n. 2, p. 133-143, 2002.

cross'

MACKAY, M. M.; METCALFE M. Multiple methods forecast for discontinuous innovations. Technological Forecasting \& Social Change. v. 69, n. 3, p. 221-232, 2002.

cross ${ }^{\text {ref }}$

MAKRIDAKIS, S.; WHEELWRIGHT, S.; HYNDMAN, R. Forecasting: Methods and Applications. 3. ed., New York: John Wiley \& Sons, 1998.

MALHOTRA, N.K. Pesquisa de marketing: uma orientação aplicada. 3. ed., Porto Alegre: Bookman, 2001.

MESQUiTA, M. A.; SANTORO, M. C. Análise de modelos e práticas de planejamento e controle de produção na indústria farmacêutica. Revista Produção. v. 14, n.1, p. 64-77, 2004.

SANDERS, N. R.; RITZMAN, L. P. Judgmental adjustment of statistical forecasts. In: ARMSTRONG, J. Principles of forecasting: a handbook for researchers and practitioners. Boston: Kluwer Academic Publishers, 2001.

SANDERS, N. R.; RITZMAN, L. P. Bringing judgment into combination forecasts. Journal of Operations Management. v. 13, n. 4, p. 311-321, 1995.

cross ${ }^{\text {ref }}$

SOUDER, W.; THOMAS, R. Significant issues for the future of product innovation. The Journal of Product Innovation Management. v. 11, n. 4, p. 344-353, 1994.

cross ${ }^{\text {ref }}$

WEBBY, R.; O’CONNOR, M. Judgmental time-series forecasting using domain knowledge. In: ARMSTRONG, J. Principles of forecasting: a handbook for researchers and practitioners. Boston: Kluwer Academic Publishers, 2001.

WEBBY, R.; O'CONNOR, M. Judgmental and statistical time series forecasting: a review of the literature. International Journal of Forecasting. v. 12, n. 1, p. 91-118, 1996.

cross'ref

WRIGHT, G.; LAWRENCE, M.; COLLOPY, F. The role and validity of judgment in forecasting. International Journal of Forecasting. v. 12, n. 1, p. 1-8, 1996.

crossef

WRIGHT, J.; Giovinazzo, R. Delphi - uma ferramenta de apoio ao planejamento prospectivo. Caderno de Pesquisas em Administração. São Paulo, v.1, n. 12, p. 54-65, 2000.

Nome completo: Fernando de Oliveira Lemos

Filiação institucional: Pontifícia Universidade Católica do Rio Grande do Sul

Departamento: Faculdade de Engenharia

Função ou cargo ocupado: Professor e Coordenador do Curso de Graduação em Engenharia de Produção

Endereço completo para correspondência (bairro, cidade, estado, país e CEP): Av. Ipiranga 6681 Prédio 30 - Secretaria, Porto Alegre, RS, Brasil, CEP 90619-900. 
Telefones para contato: (51) 33203648 R:4129

e-mail: fernando.lemos@pucrs.br

Nome completo: Flávio Sanson Fogliatto

Filiação institucional: Universidade Federal do Rio Grande do Sul

Departamento: Departamento de Engenharia de Produção

Função ou cargo ocupado: Professor do Curso de Graduação, Professor e Coordenador do curso de Pós-graduação em Engenharia de Produção

Endereço completo para correspondência (bairro, cidade, estado, país e CEP): Av. Osvaldo Aranha, $99-5^{\circ}$ andar, Porto Alegre, RS, 90035-190

Telefones para contato: +555133083491

e-mail: ffogliatto@producao.ufrgs.br 